\title{
Computational Model for Cardinality Bounded Multiset Space
}

\author{
Ibrahim Adeku Musa, Ezugwu El-Shamir Absalom and Abdulsalami Aminu \\ Department of Mathematics, Ahmadu Bello University, Zaria, Nigeria \\ Email: \{adekubash, code_abs, abdulsalami\}@yahoo.com
}

\begin{abstract}
In [1], manual generation of the elements of multiset space and mathematical models for computing the corresponding frequency numbers were presented. These approaches are quite tedious and amenable to mistakes. In this paper, we develop and implement an efficient algorithm to generate the elements of a cardinality bounded multiset space $X^{n}(m)$ for $X$ and the frequency tables for various values of $n$ and $m$. Similarly, graphical representations of the respective output tables are plotted to depict the behavioural patterns of $X^{n}(m)$ for some finite values of $n$ and $m>3$.
\end{abstract}

Keywords: Multiset, multiset space, frequency number, cardinality, algorithm.

\section{The Concept of a Multiset}

A multiset (mset for short) is a collection of objects in which those objects have multiple occurrences. A finite mset over a set $X$ is an mset $\mathrm{M}$ formed with finitely many elements from $X$ such that each element has a finite multiplicity of occurrence in $M$. Also see [2], [3], and [4] for more details.

A multiset can be represented in several ways. The use of square brackets to represent a multiset is quasi-general. Thus, a multiset containing one occurrence of a, two occurrences of $\mathrm{b}$, and three occurrences of $\mathrm{c}$ is notationally written as $[[a, b, b, c, c, c]]$ or $[a, b, b, c, c, c]$ or $[\mathrm{a}, \mathrm{b}, \mathrm{c}]_{1,2,3}$ or $[a, 2 b, 3 c]$ or $[a .1, b .2, c .3]$ or $[1 / a, 2 / b, 3 / c]$ or $\left[a^{1}, b^{2}, c^{3}\right]$ or $\left[a^{1} b^{2} c^{3}\right]$. For convenience, the curly brackets are used in place of the square brackets. In fact, the last form of representation as a string, even without using any brackets, turns out to be the most compact one, especially in computational parlance. The following schematic representation of a multiset as a numeric valued or count function abounds, particularly in the foundational development of multiset theory and its application [5][6][7].

This paper is organized as follows. In section 2 we collect preliminaries and basic definitions based on msets space and some related notions. In sections 3, a mathematical model for computing the frequency number of multiset space is presented. In section 4 , we extend mset theoretic results obtained in [1] for generating $X^{n}(m)$ values for $m \leq 3$ to $m>3$, by means of computational model. 


\section{Multiset Space and Some Related Notions}

Let $X$ be a finite set with cardinality $m$, usually called an m-mset. Let $X^{n}(m)$ denote the set of all multisets each having $m$ objects occurring with multiplicities at most $n$ times, including 0 . We call $X^{n}(m)$, a cardinality bounded multiset space for $X$ [1].

Let $X=\left\{a_{1}, a_{2}, a_{3}, \ldots, a_{m}\right\}$ be an ordered $m$-set and let $X^{n}(m)$ be a cardinality bounded space. Let an arbitrary element of $X^{n}(m)$ be denoted by $X_{<p_{i}}>$ where $\left.<p_{i}\right\rangle$ is an ordered $m$-tuple, $p_{i}$ is the multiplicity of $i^{\text {th }}$ object in $X, 1 \leq i \leq m$ and $0 \leq p i \leq n$. For convenience $X_{<p i>}$ will be denoted by $X_{p i}$. Following the aforementioned notation, the term $X_{p}$ of $X^{n}(m)$ would mean that all its objects have the same multiplicity $p$, that is $X_{p}$ is a regular mset. Also, $X_{0}$ will refer to the empty multiset or the origin of $X^{n}(m)$. It is easy to see that $X^{n}(m)$ will have $(n+1)^{m}$ elements where $n$ and $m$ are positive integers.

\subsection{Construction of $X^{n}(m)$ and their Patterns}

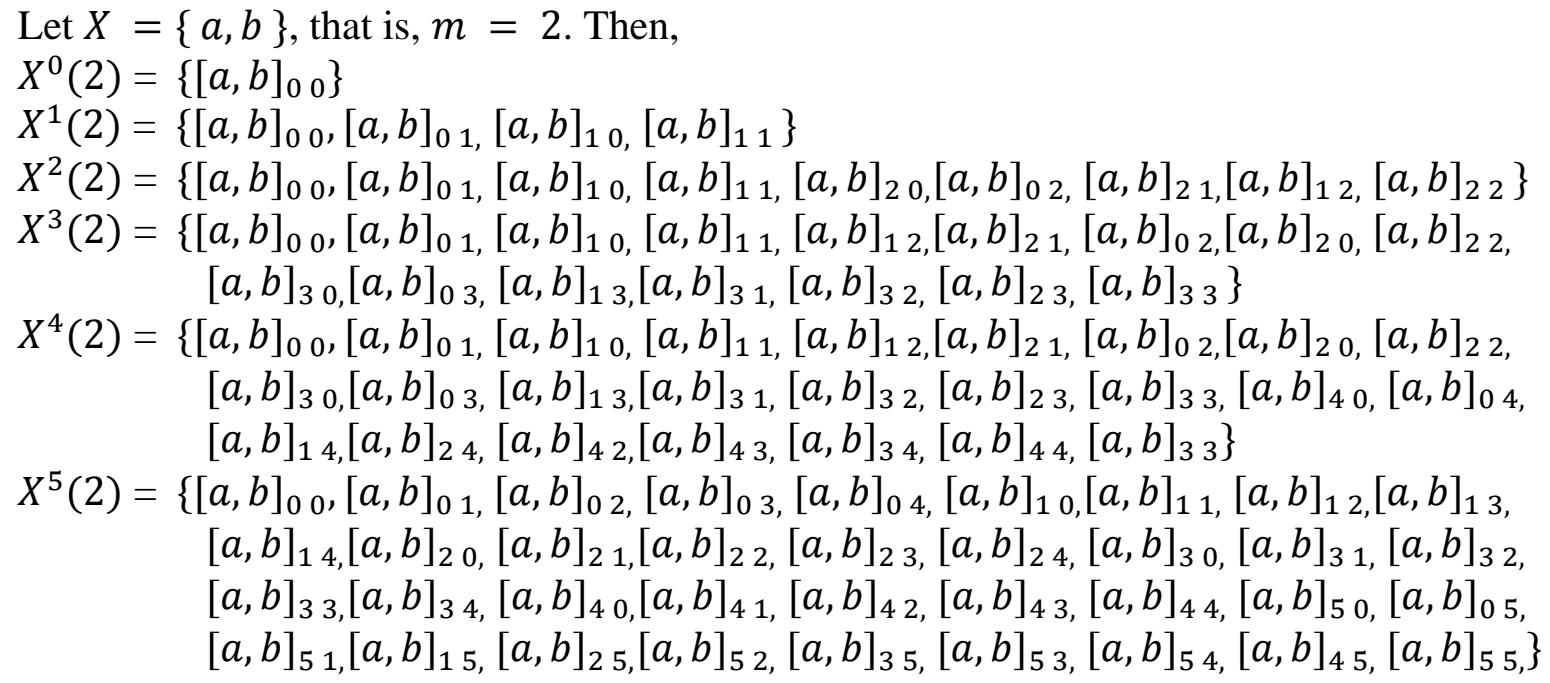

Similarly, $X^{n}(2)$ are obtained for $n=6,7,8,9, \ldots$

We take into account the cardinality of the elements of $X^{n}(m)$ to generate a pattern with reference to the frequency of their occurrences. Table I, and Table II provide a schematic representation of the said patterns for $X^{n}(2)$ and $X^{n}(3)$ for some finite values of $n$ as in [1]. Similarly, we extended this idea up to $m=4,5,6$, and 7 , that is, covering a cardinality bounded multiset space of $X^{n}(4), X^{n}(5), X^{n}(6)$, and $X^{n}(7)$ respectively, for some finite values of $n$.

Table I: Frequency value corresponding to the cardinality of the elements of $X^{n}(2)$

\begin{tabular}{|l|l|l|l|l|l|l|l|l|l|l|l|l|l|l|l|l|l|l|l|l|}
\hline & 0 & 1 & 2 & 3 & 4 & 5 & 6 & 7 & 8 & 9 & 10 & 11 & 12 & 13 & 14 & 15 & 16 & 17 & 18 \\
\hline$X^{0}(2)$ & 1 & & & & & & & & & & & & & & & & & & \\
\hline$X^{1}(2)$ & 1 & 2 & 1 & & & & & & & & & & & & & & & \\
\hline$X^{2}(2)$ & 1 & 2 & 3 & 2 & 1 & & & & & & & & & & & & & \\
\hline
\end{tabular}




\begin{tabular}{|l|l|l|l|l|l|l|l|l|l|l|l|l|l|l|l|l|l|l|l||}
\hline$X^{3}(2)$ & 1 & 2 & 3 & 4 & 3 & 2 & 1 & & & & & & & & & & & & \\
\hline$X^{4}(2)$ & 1 & 2 & 3 & 4 & 5 & 4 & 3 & 2 & 1 & & & & & & & & & & \\
\hline$X^{5}(2)$ & 1 & 2 & 3 & 4 & 5 & 6 & 5 & 4 & 3 & 2 & 1 & & & & & & & & \\
\hline$X^{6}(2)$ & 1 & 2 & 3 & 4 & 5 & 6 & 7 & 6 & 5 & 4 & 3 & 2 & 1 & & & & & & \\
\hline$X^{7}(2)$ & 1 & 2 & 3 & 4 & 5 & 6 & 7 & 8 & 7 & 6 & 5 & 4 & 3 & 2 & 1 & & & & \\
\hline$X^{8}(2)$ & 1 & 2 & 3 & 4 & 5 & 6 & 7 & 8 & 9 & 8 & 7 & 6 & 5 & 4 & 3 & 2 & 1 & & \\
\hline$X^{9}(2)$ & 1 & 2 & 3 & 4 & 5 & 6 & 7 & 8 & 9 & 10 & 9 & 8 & 7 & 6 & 5 & 4 & 3 & 2 & 1 \\
\hline
\end{tabular}

In table I, the first row $0,1,2,3, \ldots, 18$ represents the cardinality of the elements in $X^{n}(2)$ while the remaining rows represent the corresponding frequency of their occurrence. The set $X^{0}(2)$ contains only one element with cardinality zero, the set $X^{1}(2)$ contains three elements; one element with cardinality zero, two elements with cardinality one and one element with cardinality two, and so on for $n=2,3, \ldots, 9$.

It can easily be seen from the above that the number of elements in $X^{n}(m)$ is the same as the sum of the frequencies of the objects. The highest cardinality of an element $X_{p i} \in X^{n}(m)$ is $m n$ which is unique while the smallest is zero. The highest frequency of an element in $X^{n}(m)$ is $(n+1)$ and the corresponding cardinality is $n$.

Likewise, by adopting the same rigorous method of manually computing the values of $X^{n}(m)$ for cardinality $m=2$, we can also generate $X^{n}(m)$, for cardinality $m=3$ as shown below:

Let $m=3$, then we compute $X^{n}(m)$ for $n=0,1,2, \ldots, 9$.

Let $X=\{a, b, c\}$, that is, $m=3$. Then,

$X^{0}(3)=\left\{[a, b, c]_{0} \quad 00\right\}$

$X^{1}(3)=\left\{[a, b, c]_{000},[a, b, c]_{001},[a, b, c]_{010},[a, b, c]_{011}\left\{[a, b, c]_{100},[a, b, c]_{101}\right.\right.$ $\left.[a, b, c]_{110,}[a, b, c]_{111}\right\}$

$X^{2}(3)=\left\{[a, b, c]_{000},[a, b, c]_{001},[a, b, c]_{10},[a, b, c]_{010},[a, b, c]_{011},[a, b, c]_{0111}\right.$,

$[a, b, c]_{101,}[a, b, c]_{11_{0},}[a, b, c]_{111},[a, b, c]_{002},[a, b, c]_{012},[a, b]_{020}$,

$[a, b]_{021,}[a, b, c]_{0222},[a, b, c]_{120,}[a, b, c]_{200},[a, b]_{210,}[a, b]_{201}$

$[a, b, c]_{102,}[a, b, c]_{121},[a, b, c]_{112,}[a, b, c]_{122,}[a, b, c]_{200},[a, b]_{210,}[a, b]_{201}$,

$\left.[a, b, c]_{211,}[a, b, c]_{202},[a, b, c]_{220,}[a, b, c]_{212},[a, b, c]_{221,}[a, b]_{210,}[a, b]_{222,}\right\}$

Table II: Frequency value corresponding to the cardinality of the elements of $X^{n}(3)$

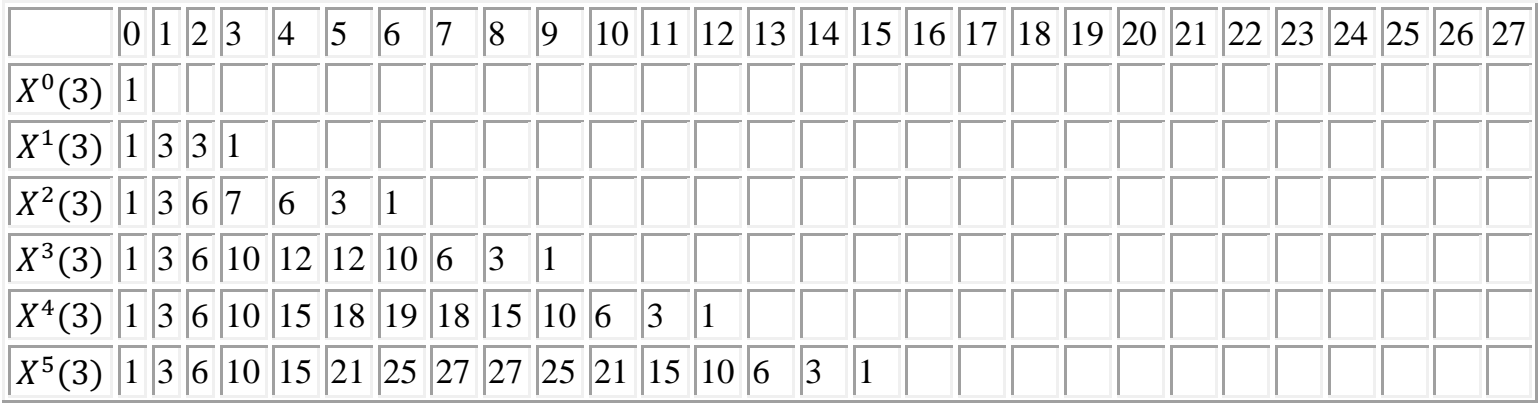




\begin{tabular}{|c|c|c|c|c|c|c|c|c|c|c|c|c|c|c|c|c|c|c|c|c|c|c|c|c|c|}
\hline$X^{6}(3)$ & \begin{tabular}{l|l|l|l|}
1 & 3 & 6 & 10
\end{tabular} & \begin{tabular}{l|l|l|}
0 & 15 & 21
\end{tabular} & 28 & 33 & $36 \mid$ & \begin{tabular}{l|l}
37 & 38
\end{tabular} & \begin{tabular}{l|l}
36 & 33
\end{tabular} & $3 \mid 28$ & 21 & 15 & 10 & 6 & 3 & 1 & & & & & & & & & & & \\
\hline$X^{7}(3)$ & $\begin{array}{llll}1 & 3 & 6 & 10\end{array}$ & \begin{tabular}{l|l|l|}
0 & 15 & 21
\end{tabular} & & 36 & 42 & \begin{tabular}{|l|l|}
46 & 48 \\
\end{tabular} & \begin{tabular}{l|l}
18 & 48
\end{tabular} & 846 & $\begin{array}{l}5 \quad 42 \\
42\end{array}$ & 36 & 28 & 321 & 11 & & 0 & 6 & 3 & 1 & & & & & & & \\
\hline$X^{8}(3)$ & \begin{tabular}{l|l|l|l}
1 & 3 & 6 & 10
\end{tabular} & $\begin{array}{l}0|15| 21 \mid \\
0\end{array}$ & 28 & $|36|^{2}$ & $45 \mid$ & $52 \mid 5$ & 5760 & $\begin{array}{lll}0 & 61\end{array}$ & 160 & $\begin{array}{l}0.57 \\
\end{array}$ & 52 & $2 \mid 45$ & $5 \mid 3$ & & 28 & 21 & 15 & 10 & 0 & & 3 & 1 & & & \\
\hline$X^{9}(3)$ & \begin{tabular}{ll|ll}
1 & 3 & 6 & 10
\end{tabular} & \begin{tabular}{l|l|l|}
0 & 15 & 21
\end{tabular} & 28 & 36 & 45 & \begin{tabular}{l|l}
55 & 6.
\end{tabular} & 6369 & 973 & $\begin{array}{ll}3 & 75 \\
\text {. }\end{array}$ & 575 & 73 & 369 & $\begin{array}{l}96 \\
6\end{array}$ & & 55 & 45 & 36 & 28 & & & 15 & & & 3 & 1 \\
\hline
\end{tabular}

In table II, The set $X^{0}(3)$ contains only one element with cardinality zero, just as in table I, while the set $X^{1}(3)$ contains four elements; one element with cardinality zero, three elements with cardinality one, three element with cardinality two and one element with cardinality three and so on.

Considering the tediousness encountered by ways of manually computing the various values of $X^{n}(n)$ with respect to the value of $m=2$ and 3. A computer program was written based on the implementation of the algorithms presented in section 5. It was observed that the program was able to compute for $X^{n}(m)$ with respect to the varying values of $n$ and $m$ within an estimated time frame.

\subsection{Construction of $X^{n}(m)$ with Varying Values of $m$}

When $m>3$ the manual computation of $X^{n}(m)$ becomes tedious and unfriendly. In this paper, we develop an algorithm with subsequent program to compute $X^{n}(m)$ and generate the frequency tables. The extended steps taken can be described as follow:

Let $X=\{a, b, c, d\}$, and $m=4$, then $X^{n}(4)$ is computed for $n=0,1,2, \ldots, 9$. with cardinality values ranging from $0,1,2, \ldots, 36$. Also for $X=\{a, b, c, d, e\}$, and $m=5$, $X^{n}(5)$ is likewise computed for $n=0,1,2, \ldots, 9$. with cardinality values ranging from $0,1,2,3, \ldots 40$, and so on for $X=\{a, b, c, d, e, f\}$ and $X=\{a, b, c, d, e, f\}$, The output generated from the computer program is as shown in tables III, IV, V, and VI below.

\subsection{Mathematical Models for Computing the Frequency Number of $m$ set Space}

In [1], equations (1) and (2) were presented as a mathematical model for computing the frequency number of mset space where it was noted that there is no generalization model for computing $f X^{n}(m)$. However, we adopted the same mathematical concept to design a computational model for obtaining the frequency number for $X^{n}(m)$, denoted by $f X^{n}(m)$ using the same recurrence formula.

Let consider some few case of how the recurrence formula can be used to generate the frequency numbers $f X^{n}(m)$ for when $m=2$ and $m=3$. A good instance of this is achieved by considering the frequency number $f X^{n}(2)$, and $f X^{n}(3)$, now by using the recurrence formula we have

$$
f X^{n}(2)=\left\{\begin{array}{cl}
\{k+1\}, & 0 \leq k \leq n \\
2 n-k+1, & n+1 \leq k \leq 2 n
\end{array}\right.
$$

Similarly, for $m=3$, the frequency number of $X^{n}(3)$, which s denoted by $f X^{n}(3)$, is given by 


$$
f X^{n}(3)= \begin{cases}\sum_{i=1}^{k}(i+1), & 0 \leq k \leq n \\ \sum_{i=1}^{n}(i+1)+\sum_{i=n+1}^{k}(3 n-2 i+1), & n+1 \leq k \leq 2 n \\ \sum_{i=1}^{n}(i+1)+\sum_{i=n+1}^{2 n}(3 n-2 i)+\sum_{i=2 n+1}^{k}(i-3 n-2), & 2 n+1 \leq k \leq 3 n\end{cases}
$$

\section{Computational Model for Generating the Frequency Number of a Multiset Space}

\section{Algorithm 1 Cardinality computation}

1. input: multiplicity $n$, cardinality $m$

2: function generateCardinality $(\mathrm{y}, \mathrm{z})$ begin

3: $n$ : multiplicity of occurrence of an object $m$ with incremental value of +1 ;

4: $\mathrm{y}$ : incremental value of $\mathrm{n}$;

5: z: function value; $X^{n}(m)$;

6: cardinailty_array $=$ array ()$; \quad$ //Array keeping cardinality of values

7: $\quad$ for $0 \leq \mathrm{a}<\mathrm{z}$ do

8: $\quad \mathrm{b} \leftarrow \operatorname{merge}(\mathrm{b}, \mathrm{y}) \quad / /$ concatenate the value of $\mathrm{b}$ and $\mathrm{y}$

9: $\quad \mathrm{a} \leftarrow \mathrm{a}+1$

10: $\quad$ end for

11: $\quad \mathrm{n} \leftarrow \mathrm{y}+1$

12: $\quad \mathrm{i} \leftarrow$ base_convert $(0, \mathrm{n}, 10)$

13: $\quad \mathrm{j} \leftarrow$ base_convert $(\mathrm{b}, \mathrm{n}, 10)$

14: $\quad \mathrm{k} \leftarrow 0$;

15: $\quad$ while $\mathrm{i} \leq \mathrm{j}$ do

16: $\quad$ res $\leftarrow$ base_convert $(\mathrm{i}, 10, \mathrm{n})$

17: $\quad$ cardinailty_array ()$\leftarrow$ res

18: $\quad \mathrm{i} \leftarrow \mathrm{i}+1$

19: $\quad$ end while

20: //Returns an array containing cardinality of values

21: return cardinailty_array()

22: end function generateCardinality

\section{Algorithms 2 computation of cardinality summation}

1: function getCardinalitySummation(cardinailty_array()) begin

2: for each cardinailty_array() as val $\boldsymbol{d o}$

3: //Tokenize digits into array

4: $\quad \mathrm{b} \leftarrow \operatorname{array}() / /$ Initialize array

5: $\quad \mathrm{c} \leftarrow 0 / /$ Initialize summation

6:

7: $\quad$ for $0 \leq \mathrm{a}<$ strlen(val) $d o$

7: $\quad \mathrm{b}[] \leftarrow \operatorname{intval}(\operatorname{substr}(\operatorname{val}, \mathrm{a}, 1))$ 
8: $\quad \mathrm{a} \leftarrow \mathrm{a}+1$

9: $\quad$ end for

10: //Sums array values

11:

12: for each b as new_val do

13: $\quad \mathrm{c} \leftarrow \mathrm{c}+$ new_val

14: end for

15:

16: //Pushes sum into new array

17: $\quad$ summ_array[] $\leftarrow$ c

18: end for

19: return summ_array()

20: end function getCardinalitySummation

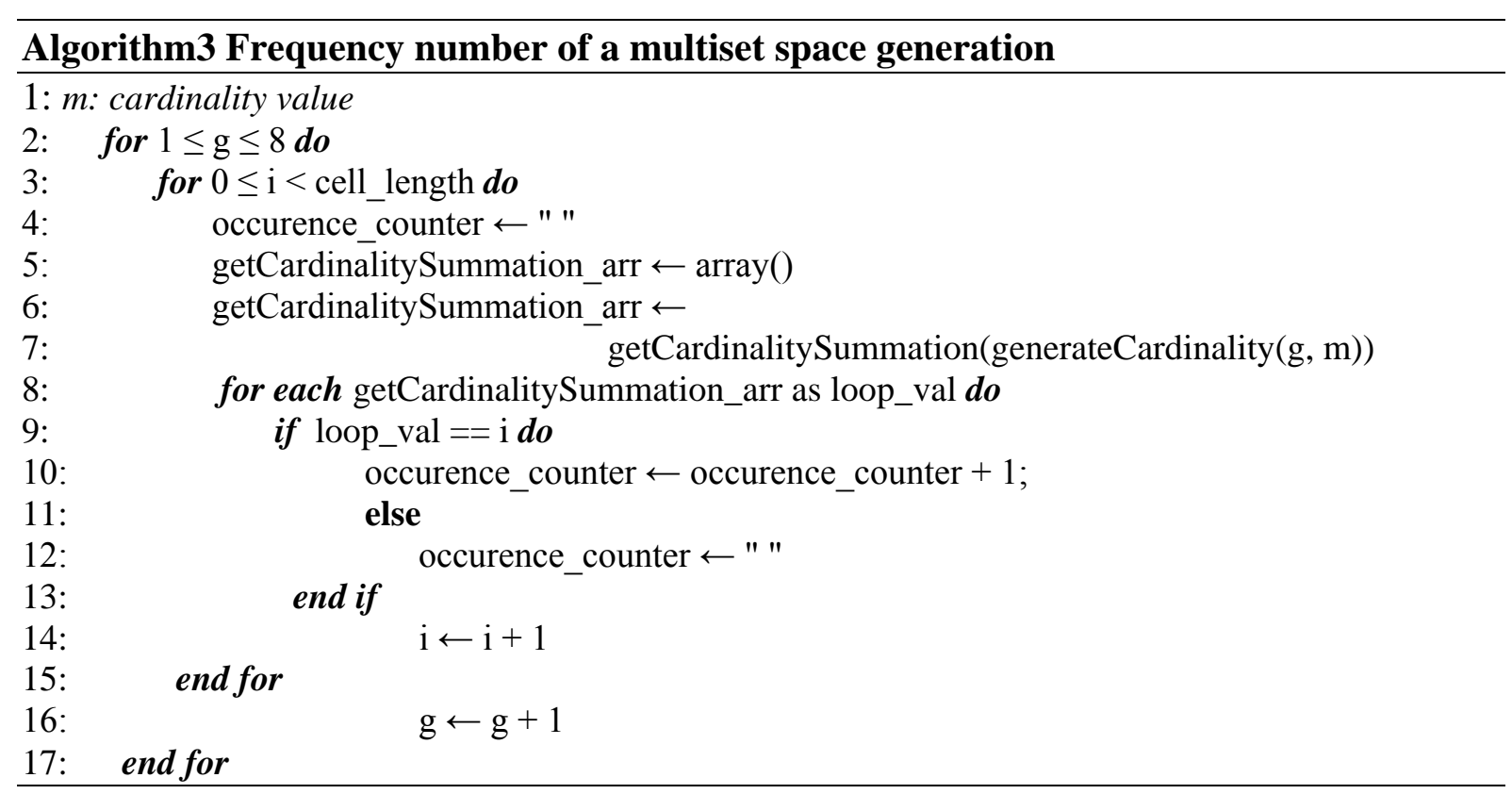

This algorithm has been implemented into a corresponding applet program named mset.java, its takes two arguments, $n$ and $m$. As an example of it use, we computed some instances of $X=\{a, b\}, X=\{a, b, c\}, X=\{a, b, c, d\}, X=\{a, b, c, d, e\}, X=\{a, b, c, d, e, f\}$, that is $m=2,3, \ldots, 6$ for $n=0,2,3, \ldots, 9$.

\section{Conclusion and Further Direction}

This study has been carried out as a result of the promising application interests eminent with the introduction of the cardinality bounded multiset space. There are major fields in computer science, for which this area is considered possible areas of application, such as data mining, search optimization techniques and conceptual proving of some program termination. Some other possible areas of application of mset theory and concepts as a whole has been studied in detail and presented in [8][9]. Most importantly, we have been able to use a computer program to generate $X^{n}(m)$ for varying values of $n$ and $m$. However, there has been no generalization case of mathematical model covering the computation of cardinality bounded mset space. We therefore present this issue as an open research topic that deserve full attention. 


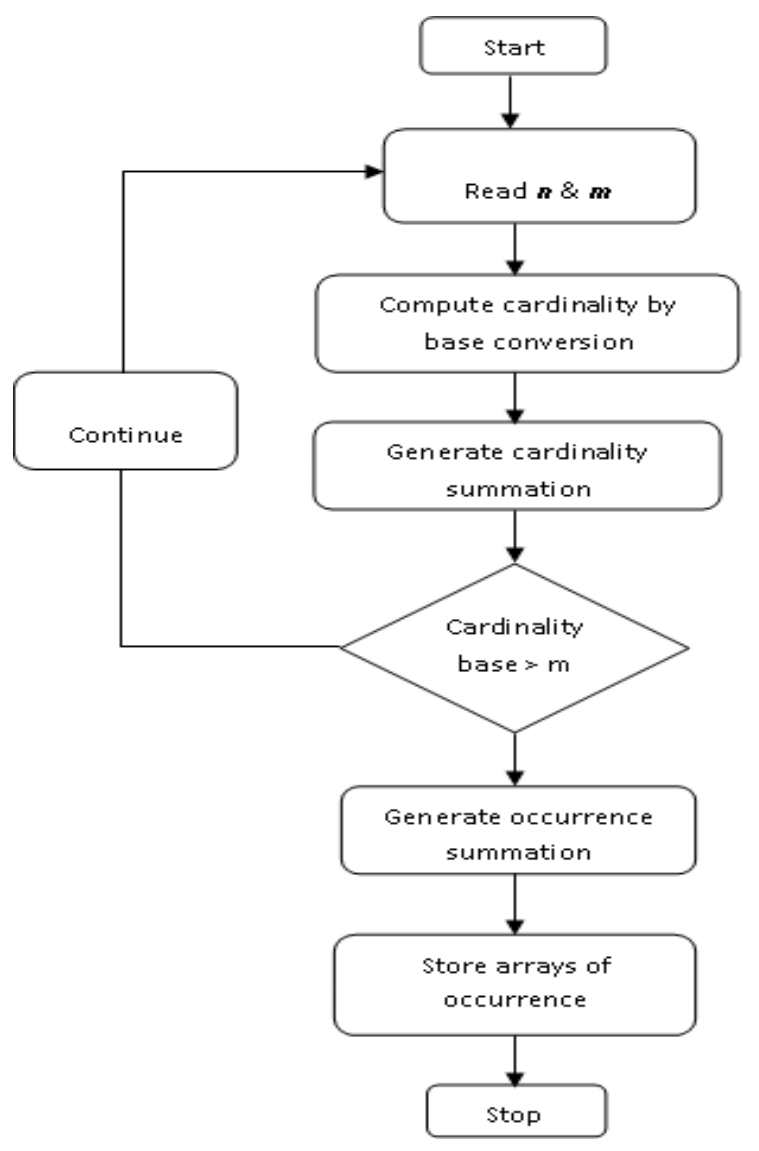

Figure 1: Program flow for the $X^{n}(m)$ computation

\section{References}

[1] A. M. Ibrahim and D. Singh, "An Outline of Multiset Space Algebra," International Journal of Algebra, Vol. 5, 2011, No. 31, 1515 - 1525.

[2] D. Singh, A. M. Ibrahim, Y. Tella, J. N. Singh, "Fundamentals of Multisets and their Applications," Novi Sad J. Math, 2007, Vol. 37, pp. 73.

[3] K. P. Girish, J. J. Sunil, "General relations between partially ordered multisets and their chains and antichains" Mathematical Communications 193 Math. Commun. Vol. 14, No. 2, 2009, pp. 193-205.

[4] D. Singh, A. M. Ibrahim, T. Yohanna, and J. N. Singh, "A Systematization of Fundamentals of Multisets," Lecturas Matem'aticas, Vol. 29, p. 5-16, 2008.

[5] D. Singh C.M. Peter, "Multiset-based Tree Model for Membrane Computing," Computer Science Journal of Moldova, vol.19, no.1 (55), 2011.

[6] T. Wieder, "Generation of All Possible Multiselections from a Multiset," Progress in Applied Mathematics Vol.2 No.1, 2011, pp. 61-66.

[7] D. Singh, A. M. Ibrahim, "Complementation in Multiset Theory," International Mathematical Forum, Vol. 6, 2011, no. 38, 1877 - 1884.

[8] D. Singh, A. M. Ibrahim, Y. Tella, J. N. Singh, "An Overview of the Applications of Multisets," Novi Sad J. Math.Vol. 37, No. 2, 2007, 73-92.

[9] A. B. Petrovsky, "Cluster Analysis in Multiset Spaces," Lecture Notes in Informatics, 2003, P-30, 109-119. 
Table III: Frequency value corresponding to the cardinality of the elements of $X^{n}(4)$

\begin{tabular}{|c|c|c|c|c|c|c|c|c|c|c|c|c|c|c|c|c|c|c|c|c|c|c|c|c|c|c|c|c|c|c|c|c|c|c|c|}
\hline & \begin{tabular}{l|l}
0 & 1
\end{tabular} & \begin{tabular}{l|l}
1 & 2
\end{tabular} & 3 & 4 & 5 & 6 & 7 & 8 & & 9 & 10 & 11 & 12 & 13 & 14 & 15 & 16 & 17 & 18 & 19 & 20 & 21 & 22 & 23 & 24 & 25 & 26 & 27 & 28 & 29 & 30 & $31 \mid$ & 32 & \begin{tabular}{|l|l|l}
33 & 34 & 3
\end{tabular} & \begin{tabular}{l|l}
35 & 36
\end{tabular} \\
\hline$X^{0}(4)$ & & & & & & & & & & & & & & & & & & & & & & & & & & & & & & & & & & & \\
\hline$X^{1}(4)$ & 14 & 46 & 4 & 1 & & & & & & & & & & & & & & & & & & & & & & & & & & & & & & & \\
\hline$X^{2}(4)$ & 15 & $4 \longdiv { 1 0 }$ & 16 & 5 & $\begin{array}{l}9 \\
16\end{array}$ & \begin{tabular}{l|l}
6 & 10
\end{tabular} & 4 & 1 & & & & & & & & & & & & & & & & & & & & & & & & & & & \\
\hline$X^{3}(4)$ & 14 & $4 \quad 10$ & 20 & 531 & 14 & $\begin{array}{l}0 \\
\end{array}$ & $4 \quad 40$ & 3 & & 20 & 10 & 4 & 1 & & & & & & & & & & & & & & & & & & & & & & \\
\hline$X^{4}(4)$ & 14 & $4 \longdiv { 1 0 }$ & 20 & 35 & 55 & 268 & 80 & 8 & & 80 & 68 & 52 & 35 & 20 & 10 & 4 & 1 & & & & & & & & & & & & & & & & & & \\
\hline$X^{5}(4)$ & 14 & $4 \quad 10$ & 20 & 35 & 55 & 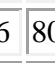 & \begin{tabular}{l|l}
0 & 10
\end{tabular} & \begin{tabular}{l|l}
4 & 12
\end{tabular} & 25 & 140 & 146 & 140 & 125 & 104 & 80 & 56 & 35 & 20 & 10 & 4 & 1 & & & & & & & & & & & & & & \\
\hline$X^{6}(4)$ & 14 & $4 \quad 10$ & 20 & 35 & 55 & \begin{tabular}{l|l}
6 & 8
\end{tabular} & \begin{tabular}{l|l}
4 & 11
\end{tabular} & \begin{tabular}{l|l}
6 & 12
\end{tabular} & 49 & 180 & 206 & 224 & 231 & 224 & 206 & 180 & 149 & 116 & 84 & 56 & 35 & 20 & 10 & 4 & 1 & & & & & & & & & & \\
\hline$X^{7}(4)$ & 14 & $4 \quad 10$ & 20 & 35 & 55 & 68 & 412 & 16 & & 204 & 246 & 284 & 315 & 336 & 344 & 336 & 315 & 284 & 246 & 204 & 161 & 120 & 84 & 56 & 35 & 20 & 10 & 4 & 1 & & & & & & \\
\hline$X^{8}(4)$ & 14 & $4 \mid 11$ & 20 & 035 & 556 & \begin{tabular}{l|l}
6 & 8
\end{tabular} & \begin{tabular}{l|l}
4 & 12
\end{tabular} & \begin{tabular}{l|l}
0 \\
0
\end{tabular} & & 216 & 270 & 324 & 375 & 420 & 456 & 480 & 489 & 480 & 456 & 420 & 375 & 324 & 270 & $\begin{array}{ll}0 & 216\end{array}$ & 165 & 120 & 84 & 56 & 35 & 20 & & 4 & 1 & & \\
\hline$X^{9}(4)$ & 14 & $4 \quad 18$ & 20 & 535 & 55 & 68 & 412 & $5 \longdiv { 1 6 }$ & 65 & 220 & 282 & 348 & 415 & 480 & 540 & 592 & 633 & 660 & 670 & 660 & 633 & 592 & 540 & 0 & 415 & 348 & 282 & 220 & 165 & 120 & 84 & 56 & 35 & $\begin{array}{lll}20 & 10 & 4\end{array}$ & 4 \\
\hline
\end{tabular}

Table IV: Frequency value corresponding to the cardinality of the elements of $X^{n}(5)$

\begin{tabular}{|c|c|c|c|c|c|c|c|c|c|c|c|c|c|c|c|c|c|c|c|c|c|c|c|c|c|c|c|c|c|c|c|c|c|c|c|c|c|c|c|c|}
\hline & \begin{tabular}{|l|l|l|l|}
0 & 1
\end{tabular} & & 3 & 4 & 5 & 6 & 7 & 8 & 9 & 10 & 11 & 12 & 13 & 14 & 15 & 16 & 17 & 18 & 19 & 20 & 21 & 22 & 23 & 24 & 25 & 26 & 27 & 28 & 29 & 30 & 31 & 32 & 33 & 34 & 35 & 36 & 37 & 38 & 39 & 40 \\
\hline$X^{0}(5)$ & 1 & & & & & & & & & & & & & & & & & & & & & & & & & & & & & & & & & & & & & & & \\
\hline$X^{1}(5)$ & 15 & $\begin{array}{ll}5 & 10\end{array}$ & 10 & & 1 & & & & & & & & & & & & & & & & & & & & & & & & & & & & & & & & & & & \\
\hline$X^{2}(5)$ & 15 & 515 & 30 & 45 & 51 & 45 & 30 & 15 & 5 & 1 & & & & & & & & & & & & & & & & & & & & & & & & & & & & & & \\
\hline$X^{3}(5)$ & 15 & 515 & 35 & 65 & 101 & 135 & 155 & 155 & 135 & 101 & 65 & 35 & 15 & 5 & 1 & & & & & & & & & & & & & & & & & & & & & & & & & \\
\hline$X^{4}(5)$ & 15 & 515 & 35 & 70 & 121 & 185 & 255 & 320 & 365 & 381 & 365 & 320 & 255 & 185 & 121 & 70 & 35 & 15 & 5 & 1 & & & & & & & & & & & & & & & & & & & & \\
\hline$X^{5}(5)$ & 15 & 515 & 35 & 70 & 126 & 205 & 305 & 420 & 540 & 651 & 735 & 780 & 780 & 735 & 651 & 540 & 420 & 305 & 205 & 126 & 70 & 35 & 15 & 5 & 1 & & & & & & & & & & & & & & & \\
\hline$X^{6}(5)$ & 15 & 515 & 535 & 570 & 126 & 210 & 325 & 470 & 640 & 826 & 1015 & 1190 & 1330 & 1420 & 1451 & 1420 & 1330 & 1190 & 1015 & 826 & $640 \mid$ & 470 & 325 & 210 & 126 & 70 & 35 & 15 & 5 & 1 & & & & & & & & & & \\
\hline$X^{7}(5)$ & 15 & 515 & 53 & 570 & 126 & 210 & 330 & 490 & 690 & 926 & 1190 & 1470 & 1750 & 2010 & 2226 & 2380 & 2460 & 2460 & 2380 & 2226 & 2010 & 1750 & 1470 & 1190 & 926 & 690 & 490 & 330 & 210 & 126 & 70 & 35 & 15 & 5 & 1 & & & & & \\
\hline$X^{8}(5)$ & 15 & $\begin{array}{ll}5 & 15\end{array}$ & 35 & 70 & 126 & 210 & 330 & 495 & 710 & 976 & 1290 & 1645 & 2030 & 2430 & 2826 & 3195 & 3510 & 3750 & 3900 & 3951 & 3900 & 3750 & 3510 & 3195 & 2826 & 2430 & 2030 & 1645 & 1290 & 976 & 710 & 495 & 330 & 210 & 126 & 70 & 35 & 15 & 5 & 1 \\
\hline
\end{tabular}


Table V: Frequency value corresponding to the cardinality of the elements of $X^{n}(6)$

\begin{tabular}{|c|c|c|c|c|c|c|c|c|c|c|c|c|c|c|c|c|c|c|c|c|c|c|c|c|c|c|c|c|c|c|c|c|c|c|c|c|c|c|c|c|c|c|}
\hline & $\sqrt{0} \sqrt{1}$ & $\sqrt{2}$ & $\sqrt{4}$ & 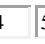 & 5 & 6 & 7 & 8 & 9 & 10 & 11 & 12 & 13 & 14 & 15 & 16 & 17 & 18 & 19 & 20 & 21 & 22 & 23 & 24 & 25 & 26 & 27 & 28 & 29 & 30 & 31 & 32 & 33 & 34 & 35 & 36 & 37 & 38 & 39 & 40 & 41 & 42 \\
\hline$X^{0}(6)$ & $\mid 1\rceil$ & & & & & & & & & & & & & & & & & & & & & & & & & & & & & & & & & & & & & & & & & \\
\hline$X^{1}(6)$ & 16 & 152 & & 15 & 6 & 1 & & & & & & & & & & & & & & & & & & & & & & & & & & & & & & & & & & & & \\
\hline$X^{2}(6)$ & 16 & 215 & & 90 & 126 & 141 & 126 & 90 & 50 & 21 & 6 & 1 & & & & & & & & & & & & & & & & & & & & & & & & & & & & & & \\
\hline$X^{3}(6)$ & 16 & 215 & & 1202 & 216 & 336 & 456 & 546 & 580 & 546 & 456 & 336 & 216 & 120 & 56 & 21 & 6 & 1 & & & & & & & & & & & & & & & & & & & & & & & & \\
\hline$X^{4}(6)$ & $\mid \sqrt{16}$ & 215 & & $\left.126\right|_{2}$ & 246 & 426 & 666 & 951 & 1246 & $\mid 1506$ & 1686 & 1751 & 1686 & 1506 & 1246 & 951 & 666 & 426 & 246 & 126 & 56 & 21 & 6 & 1 & & & & & & & & & & & & & & & & & & \\
\hline$X^{5}(6)$ & 16 & 215 & & 126 & 252 & 456 & 756 & 1161 & 1666 & $|2247|$ & 2856 & 3431 & 3906 & 4221 & 4332 & 4221 & 3906 & 3431 & 2856 & 2247 & 1666 & 1161 & 756 & 456 & 252 & 126 & 56 & 21 & 6 & 1 & & & & & & & & & & & & \\
\hline$X^{7}(6)$ & $\mid 16$ & 21 & & 126 & 252 & 462 & 792 & 1281 & 1966 & $|2877|$ & 4032 & 5432 & 7056 & 8856 & 10752 & 12642 & 14412 & 15946 & 17136 & 17892 & 18152 & 17892 & $\mid 17136$ & 15946 & 14412 & 12642 & 10752 & 8856 & 7056 & 5432 & 4032 & 2877 & 1966 & 1281 & 792 & 462 & 252 & 126 & 56 & 21 & 6 & 1 \\
\hline
\end{tabular}

Table VI: Frequency value corresponding to the cardinality of the elements of $X^{n}(7)$

\begin{tabular}{|c|c|c|c|c|c|c|c|c|c|c|c|c|c|c|c|c|c|c|c|c|c|c|c|c|c|c|c|c|c|}
\hline & 0 & 1 & 2 & 3 & 4 & 5 & 6 & 7 & 8 & 9 & 10 & 11 & 12 & 13 & 14 & 15 & 16 & 17 & 18 & 19 & 20 & 21 & 22 & 23 & 24 & 25 & 26 & 27 & 28 \\
\hline$X^{0}(7)$ & 1 & & & & & & & & & & & & & & & & & & & & & & & & & & & & \\
\hline$X^{1}(7)$ & 1 & 7 & 21 & 35 & 35 & 21 & 7 & 1 & & & & & & & & & & & & & & & & & & & & & \\
\hline$X^{2}(7)$ & 1 & 7 & 28 & 77 & 161 & 266 & 357 & 393 & 357 & 266 & 161 & 77 & 28 & 7 & 1 & & & & & & & & & & & & & & \\
\hline$X^{3}(7)$ & 1 & 7 & 28 & 84 & 203 & 413 & 728 & 1128 & 1554 & 1918 & 2128 & 2128 & 1918 & 1554 & 1128 & 728 & 413 & 203 & 84 & 28 & 7 & 1 & & & & & & & \\
\hline$X^{4}(7)$ & 1 & 7 & 28 & 84 & 210 & 455 & 875 & 1520 & 2415 & 3535 & 4795 & 6055 & 7140 & 7875 & 8135 & 7875 & 7140 & 6055 & 4795 & 3535 & 2415 & 1520 & 875 & 455 & 210 & 84 & 28 & 7 & 1 \\
\hline
\end{tabular}




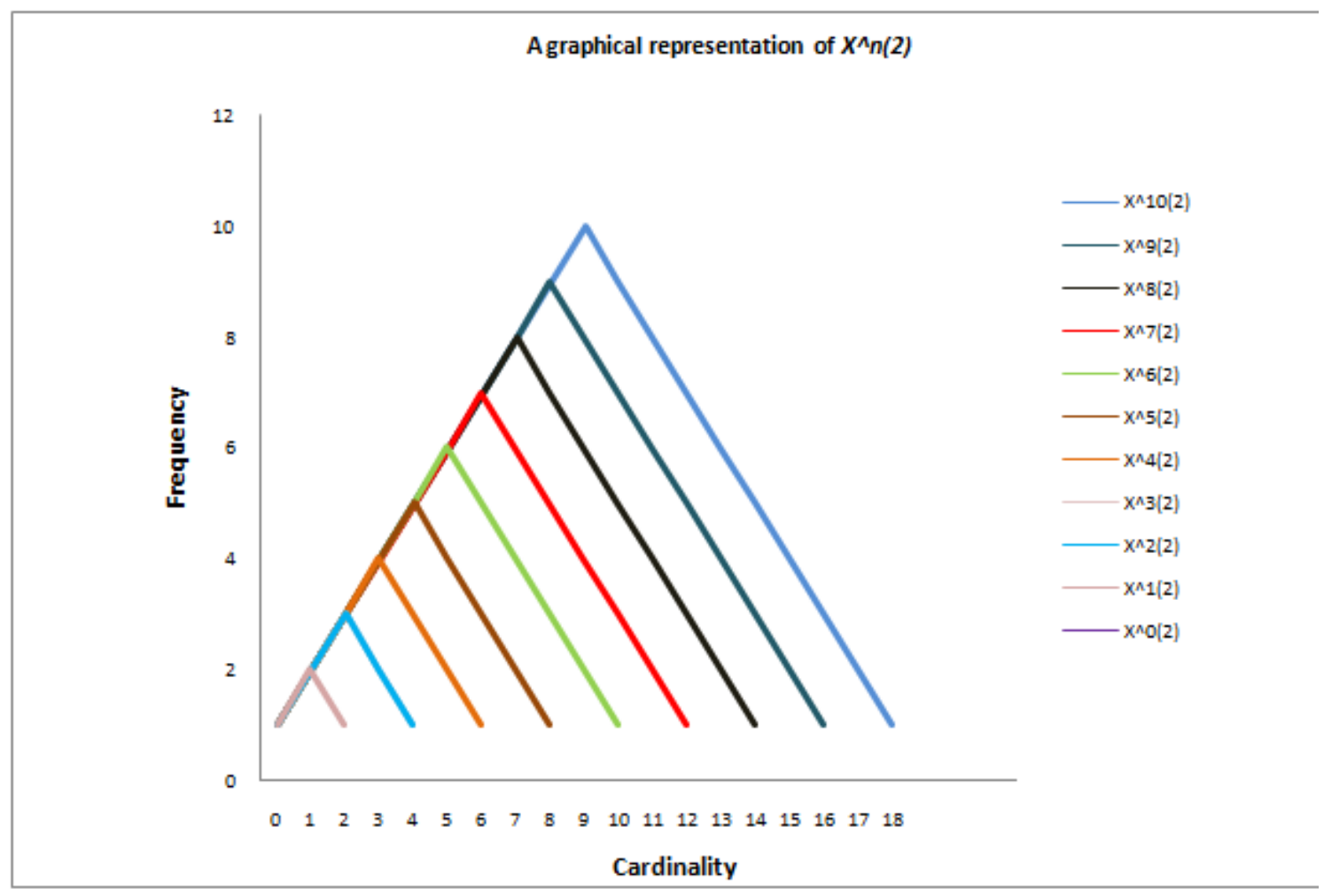

Figure 1:

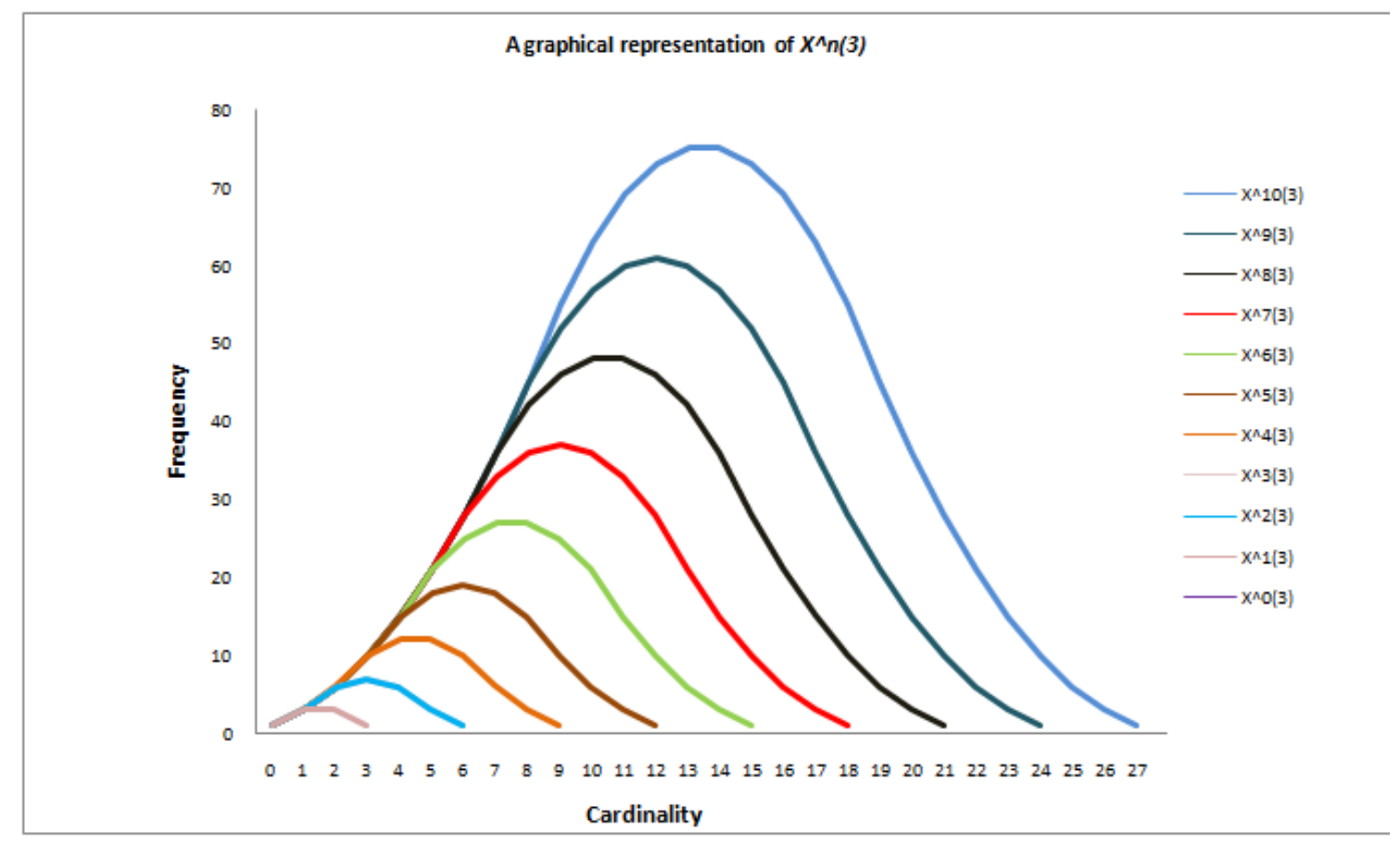

Figure 2: 


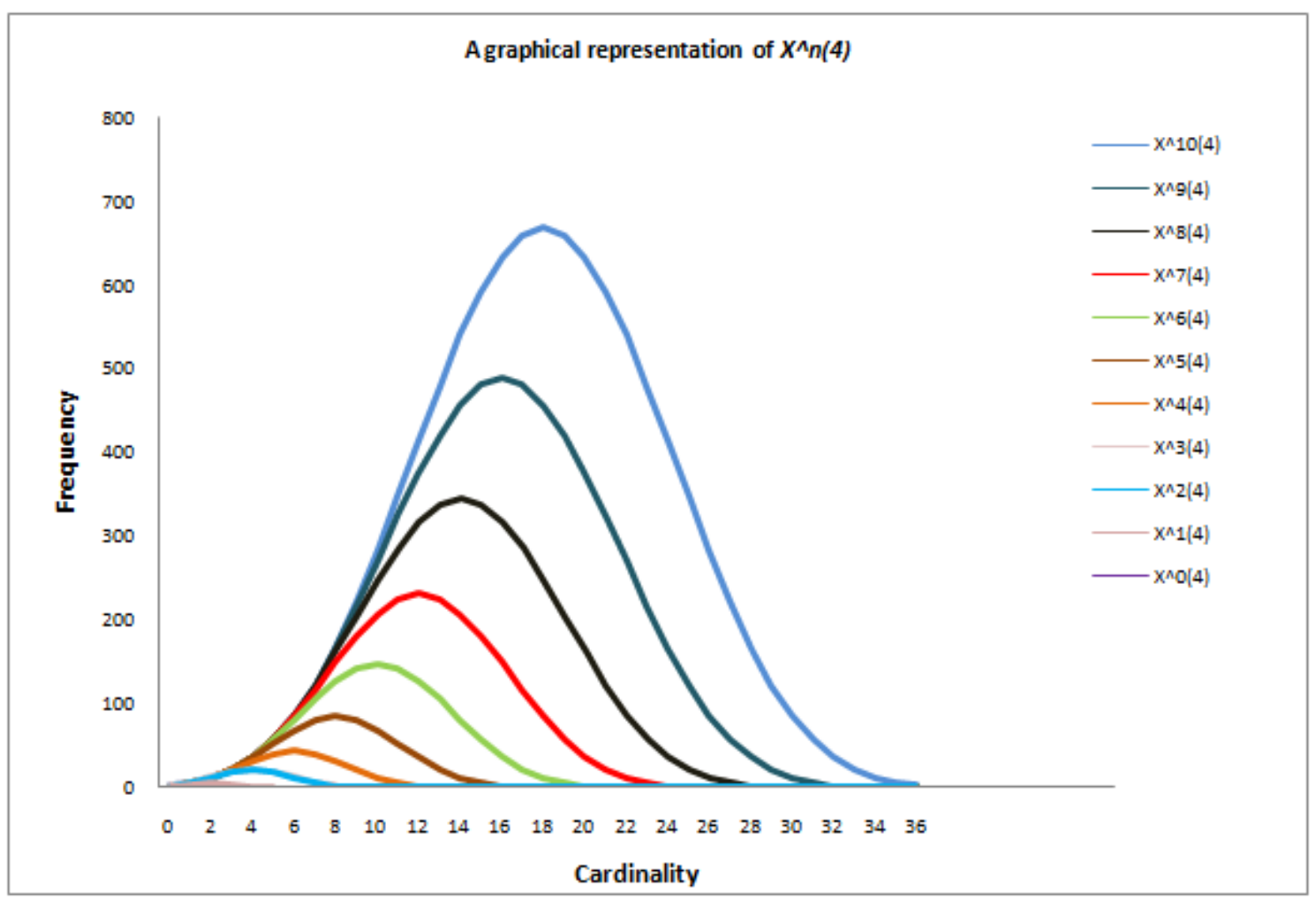

Figure3:

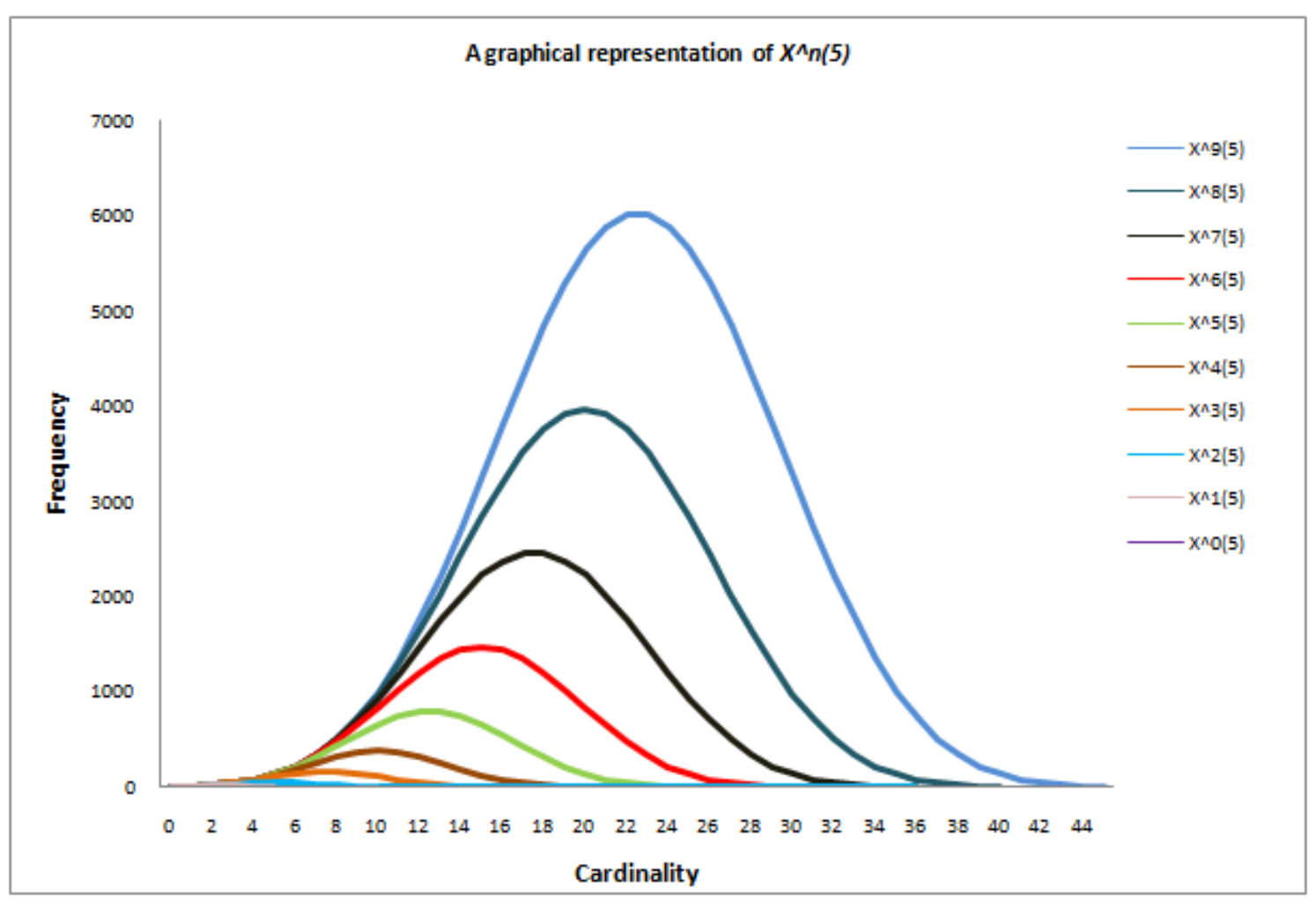

Figure 4: 


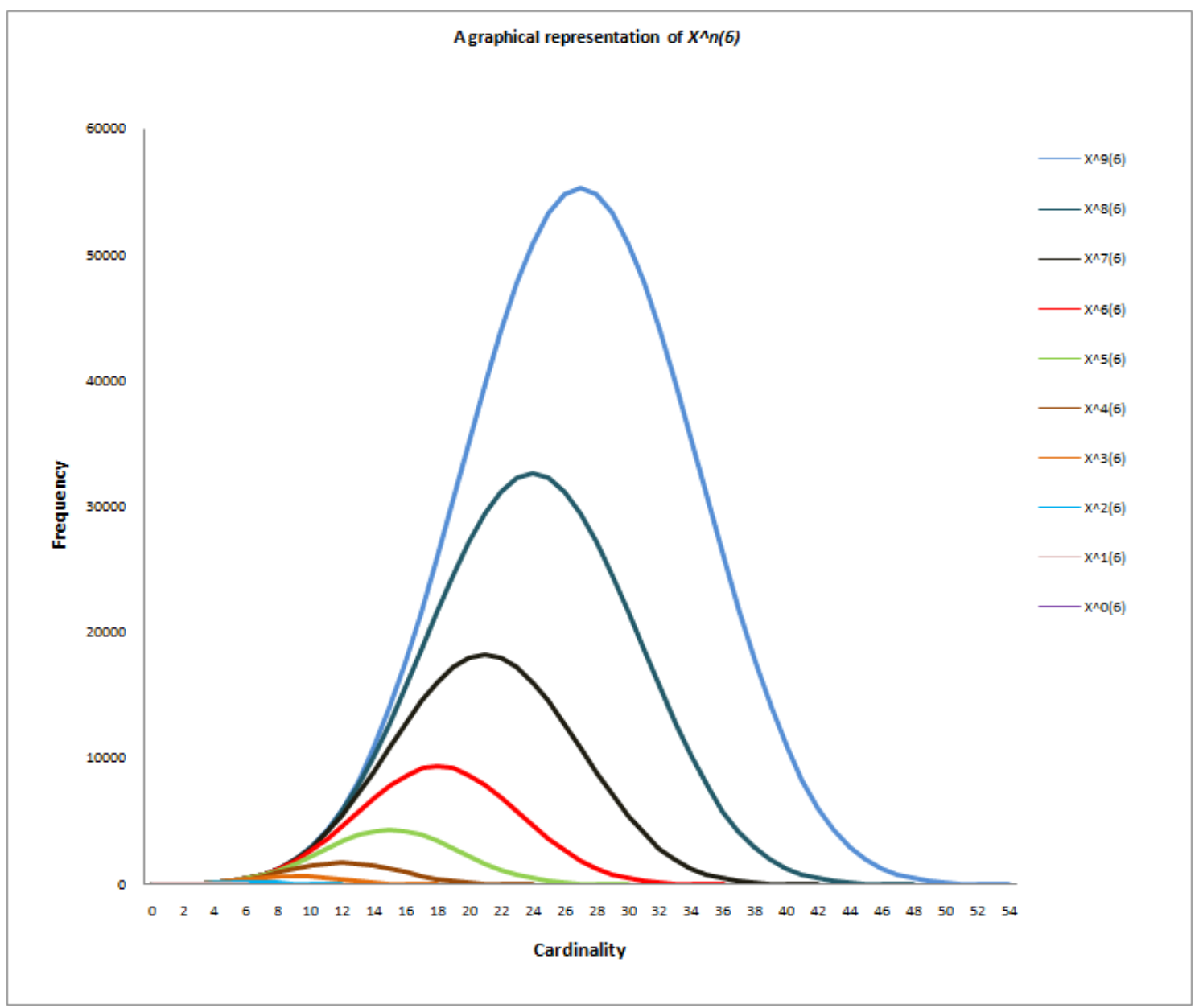

Figure 5:

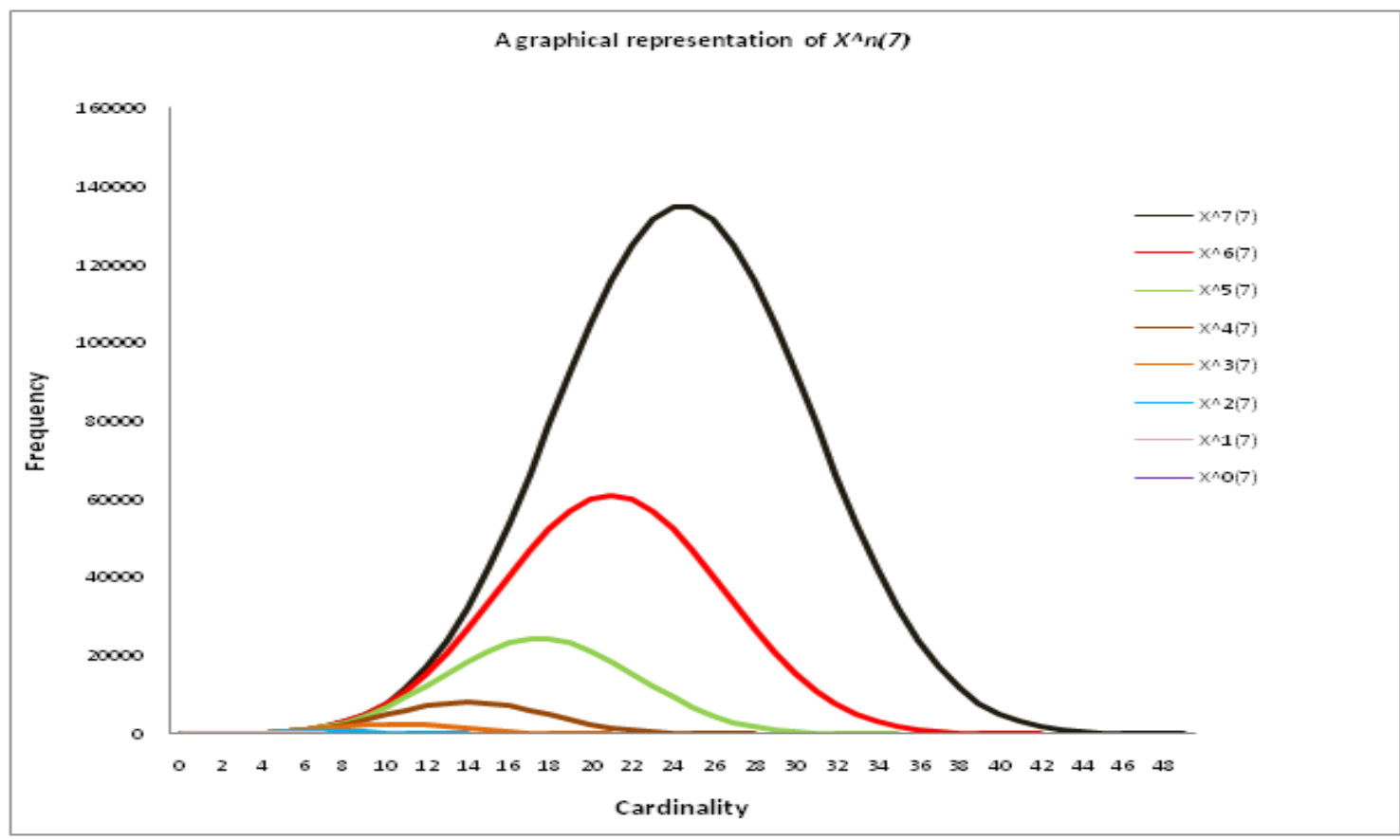

Figure 6: 